\title{
Modular Assembly of Versatile Nanoparticles with Epigallocatechin Gallate
}

Zeng Yi, a,b Guangcan Chen, ${ }^{\mathrm{a}, \mathrm{b}}$ Xiangyu Chen, ${ }^{\mathrm{a}, \mathrm{b}}$ Zhe Sun, ${ }^{\mathrm{a}, \mathrm{b}}$ Xiaomin Ma, ${ }^{\mathrm{a}, \mathrm{b}}$ Wen $\mathrm{Su},{ }^{\mathrm{a}, \mathrm{b}}$ Zhiwen Deng, ${ }^{\mathrm{a}, \mathrm{b}}$ Lei Ma, ${ }^{\mathrm{a}, \mathrm{b}}$ Yaqin Ran, ${ }^{\mathrm{a}, \mathrm{b}}$ Qiulan Tong, ${ }^{\mathrm{a}, \mathrm{b}}$ and Xudong Li * a,b

${ }^{a}$ National Engineering Research Center for Biomaterials, Sichuan University, Chengdu 610064, P. R. China.

${ }^{\mathrm{b}}$ Engineering Research Center in Biomaterials, Sichuan University, Chengdu 610064, P. R. China.

Corresponding Author

* E-mail: xli20004@yahoo.com;

Fax: +86 (0)28 85412102 ;

Tel: +86(0)28 85412102 .

Total 6 pages, 7 Figures and 1 Table

\section{Table of contents}

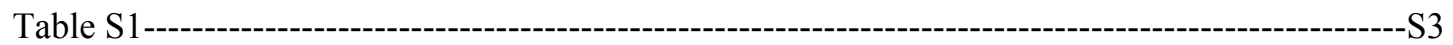

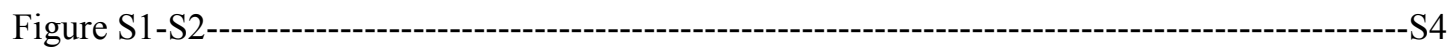

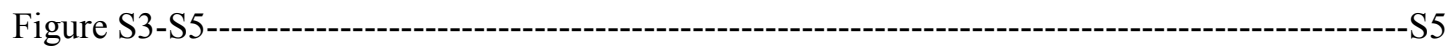

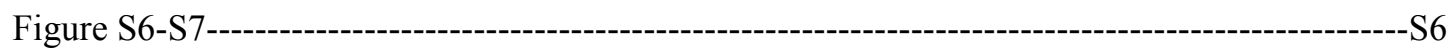


Materials. EGCG ( $>94 \%$, Taiyo Green Power Co., Ltd), resorcinol (AR, Kelong), formaldehyde (37\%, Kelong), Arg-Gly-Asp (97\%, Meilunbio), N-Boc-ethylenediamine (98\%, J\&K Scientific Ltd.), aminoacetaldehyde dimethyl acetal (98\%, J\&K Scientific Ltd.), L-lysine (98\%, J\&K Scientific Ltd.), pluronic F-127 (Sigma), fluorescein 5(6)-isothiocyanate (FITC, 90\%, Sigma), 5aminopymofluorescein (95\%, Dalian Meilun Biotechnology), N-Hydroxysuccinimide (98\%, Sigma), N-(3-Dimethylaminopropyl)-N'-ethylcarbodiimide hydrochloride crystalline (97\%, Sigma), Trifluoroacetic acid (AR, Kelong), polyethylene glycol monomethyl ether 4000 (98\%, TCI), polyethylenimine branched (average $\mathrm{Mw} 800$, Sigma), cystamine dihydrochloride (98\%, J\&K Scientific Ltd.), allylamine hydrochloride acid (98\%, TCI), propargyl amine ( $\geq 97 \%$, TCI), glycine (99\%, Amresco), urea (99\%, J\&K Scientific Ltd.), glutaraldehyde solution (25\%, Kelong), glutathione (GSH, TCI), gemcitabine (98\%, Dalian Meilun Biotechnology), gentamicin sulfate ( $>590 \mathrm{IU} / \mathrm{MG}, \mathrm{BR}$, Dalian Meilun Biotechnology), bleomycin sulfate (1.5 2.0 U/mg, BR, Dalian Meilun Biotechnology), Cell Counting Kit-8 (CCK-8, Dojindo).

Microscopy. The SEM images of the samples were observed using JSM-7500F scanning electron microscope. For TEM observations, $10 \mu \mathrm{L}$ of the diluted samples were dipped onto a formvar/carbon-coated copper grid (Electron Microscopy Sciences), and stained with $1 \%$ phosphotungstic acid, and observed by using a JEOL JEM-1200 EX microscope operating at 120 $\mathrm{kV}$.

Dynamic light scattering (DLS) and infrared spectroscopy (FT-IR). Potassium bromide (KBr) was used to disperse the lyophilized nanoparticle powders and pressed tablet. The FT-IR spectra were collected in the range of $400-4000 \mathrm{~cm}^{-1}$. The Nanosizer ZS (Malvern Instruments) was used to determine the size and polydispersity index of nanoparticles.

The ornamented nanoparticles with FITC and fluoresceinamine. The R2, R3 and R4 nanoparticles were heated to $80^{\circ} \mathrm{C}$ for $4 \mathrm{~h}$ and their protecting groups were removed using trifluoroacetic acid and hydrochloric acid, respectively. The R2 nanoparticles were dispersed in sodium bicarbonate buffer $(\mathrm{pH} 9.8)$ and mixed with FITC $(0.1 \mathrm{mg} / \mathrm{mL})$ for stirring $12 \mathrm{~h}$ in the dark. Finally, the FITC-grafted R2 nanoparticles were washed with absolute ethanol and collected by centrifugation. The $10 \mathrm{mg}$ of $\mathrm{R} 3$ nanoparticles were dispersed in ethanol, and then the solution was 
heated to reflux at $60{ }^{\circ} \mathrm{C}$ for $4 \mathrm{~h}$ after the addition of $10 \mathrm{mg} 5$-aminopymofluorescein. The fluoresceinamine-grafted R3 nanoparticles were obtained after washed with absolute ethanol and centrifugation. With the addition of $30 \mathrm{mg}$ EDC and $30 \mathrm{mg}$ NHS, $10 \mathrm{mg}$ 5-aminopymofluorescein was added into the R4 nanoparticles (10 mg) dispersed in DMSO and the mixture was stirred for 12 h. Finally, the fluoresceinamine-grafted R4 nanoparticles were washed with absolute ethanol and collected by centrifugation. The fluorescence emission ability of the ornamented nanoparticles was investigated using fluorescence microscope.

\begin{tabular}{|l|l|l|l|}
\hline Polyphenol & Amines & Aldehyde & $\begin{array}{l}\text { Abbreviation of } \\
\text { NPs }\end{array}$ \\
\hline EGCG & RGD-peptide & Formaldehyde & R1 NPs \\
\hline EGCG & $\begin{array}{l}\text { N-Boc- } \\
\text { ethylenediamine }\end{array}$ & Formaldehyde & R2 NPs \\
\hline EGCG & $\begin{array}{l}\text { Aminoacetaldehyde } \\
\text { dimethyl acetal } \\
\text { aldehyde }\end{array}$ & Formaldehyde & R3 NPs \\
\hline EGCG & Lysine & Formaldehyde & R4 NPs \\
\hline EGCG & PEG-PEI(1:1) & Formaldehyde & R5 NPs \\
\hline EGCG & PEG-PEI(5:1) & Formaldehyde & R51 NPs \\
\hline Resorcinol & Cystamine & Formaldehyde & R6 NPs \\
\hline EGCG & Allylamine & Formaldehyde & R7 NPs \\
\hline EGCG & $\begin{array}{l}\text { Allylamine } \\
\text { F127 }\end{array}$ & Formaldehyde & R71 NPs \\
\hline EGCG & Propargyl amine & Formaldehyde & R8 NPs \\
\hline EGCG & Glycine & Formaldehyde & Gly NPs \\
\hline EGCG & Glycine with F127 & Formaldehyde & \\
\hline EGCG & Cystamine & Formaldehyde & Cys NPs \\
\hline EGCG & Gemcitabine & Formaldehyde & Gem NPs \\
\hline EGCG & Bleomycin & Formaldehyde & Ble NPs \\
\hline EGCG & Gentamicin & Formaldehyde & Gen NPs \\
\hline EGCG & Urea & Formaldehyde & Urea NPs \\
\hline EGCG & Glycine & Glutaraldehyde & GPF \\
\hline & & & \\
\hline
\end{tabular}

Table S1. The abbreviation of NPs. 

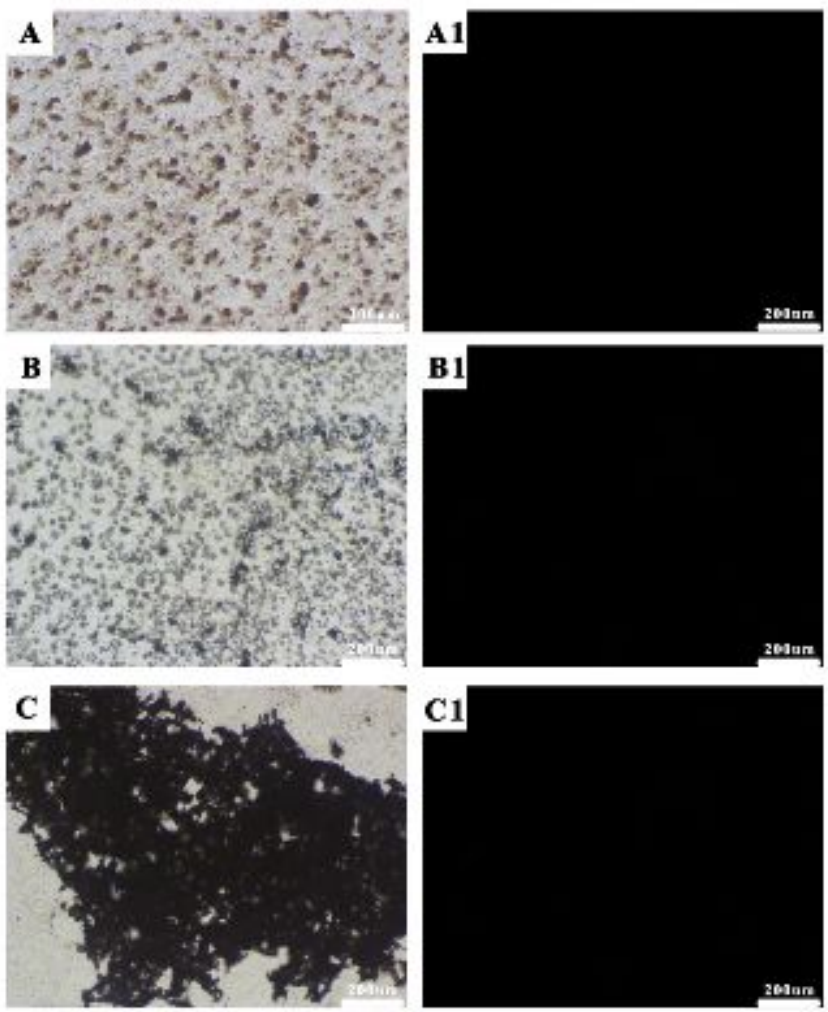

Figure S1. (A-C) Microscopy optical photoes of the nanoparticles after solidification. (A1-C1) Fluorescence photographs of solidification nanoparticles modified without fluorescence molecules at $405 \mathrm{~nm}$ excitation.

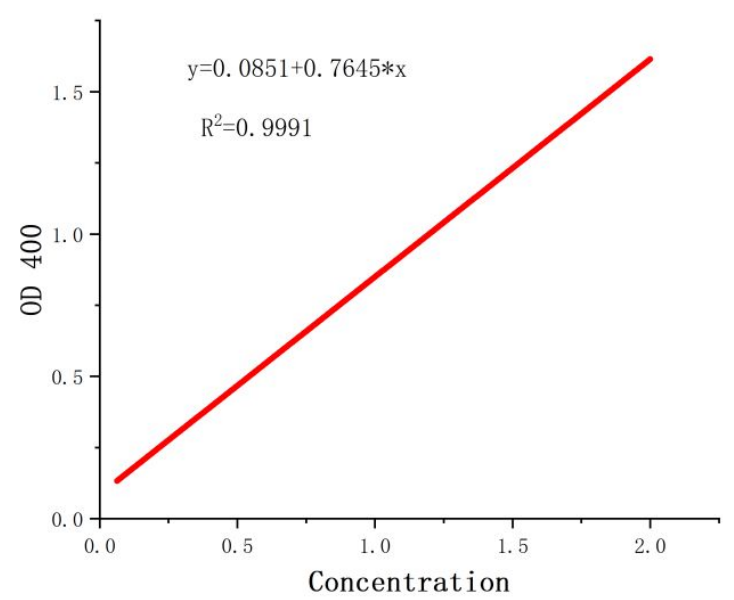

Figure S2. The standard curve of OD 400 values at different concentration of nanoparticle. 


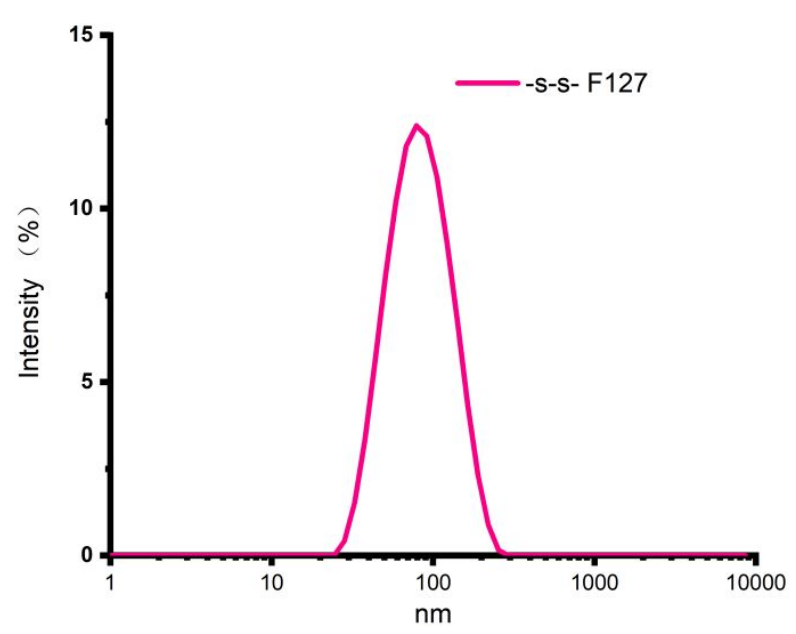

Figure S3. The DLS result of -s-s- nanoparticles synthesized by using F127.

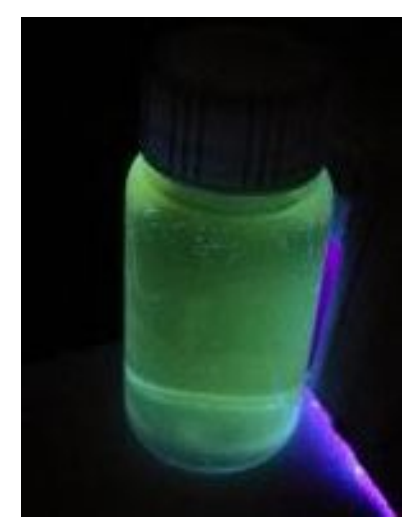

Figure S4. The photo image of fluorescence particles.

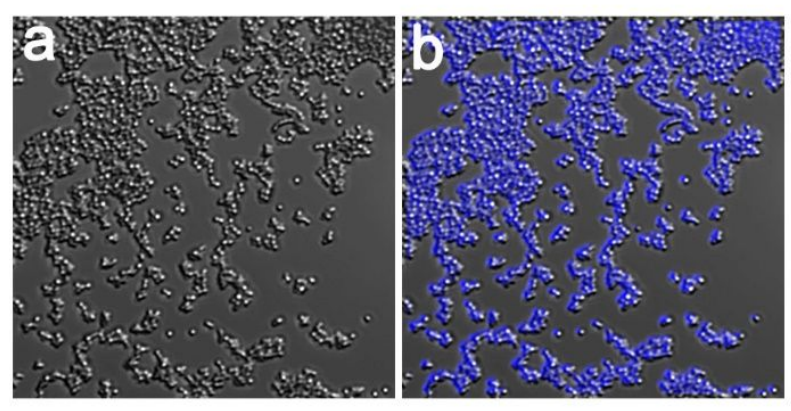

Figure S5. Images were obtained by (a) optical microscopy and (b) imaged fluorescence microscopy. 


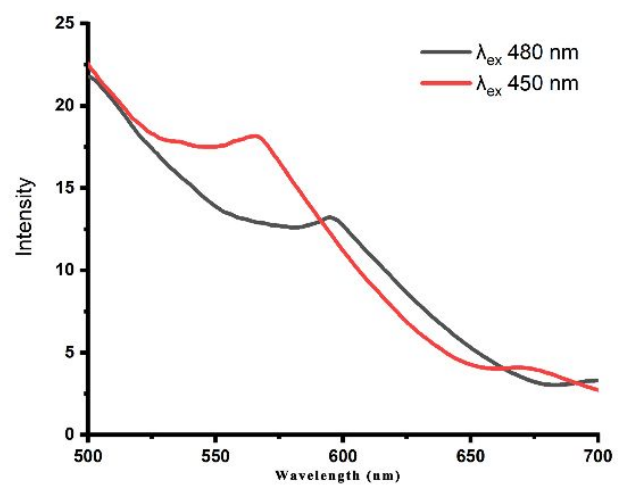

Figure S6. The fluorescent spectrum of GFP.

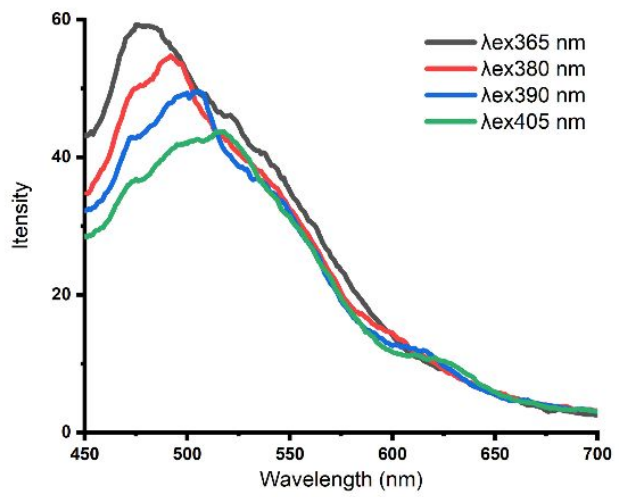

Figure S7. The fluorescent spectrum of dissolved GFP. 